\title{
Learning and transfer of oddity as a function of apparatus and trials per problem'
}

\author{
PASCHAL N. STRONG, JR. ${ }^{2}$ \\ TEXAS TECHNOLOGICAL COLLEGE
}

\begin{abstract}
Fourteen naive Rhesus monkeys were trained on one of three apparatus (The Wisconsin General Test Apparatus, the Primate Automatic Test Apparatus-Key, and the Primate Automatic Test Apparatus-Lever). The problem to be learned was a 6-trial or 1-trial, two position, oddity problem. Six-trial animals were switched to the 1-trial problem upon reaching criterion. Upon attaining the 1-trial criterion, a test series of novel problems was presented to each animal. Twelve of the 14 animals were then transferred to a new apparatus and again learned the oddity problem. The following were concluded: (1) Animals appear to learn as well with two dimensional stimuli as with three dimensional. (2) Animals reach criterion on 1-trial problems as soon as 6-trial problems. (3) Six-trial animals require a significant number of additional trials to reach the 1-trial criterion. (4) When an animal is transferred to a new apparatus and given the same oddity problems, there is no evidence of any positive transfer.
\end{abstract}

\section{Problem}

The purpose of this study was (1) to compare the learning of the oddity problem on two devices using automatically presented, three dimensional displays as contrasted with the WGTA, (2) to explore the effects of trials per problem in learning oddity, and (3) to investigate the transfer of learning from one apparatus to the other.

\section{Method}

Ss were 12 immature and two mature Rhesus monkeys.

\section{Apparatus}

The WGTA used in this study was similar in all important respects to the apparatus described by Harlow.

The Primate Automatic Testing Apparatus-Lever (PATA-L) (Rohles et al, 1961) consisted of three horizontal in-line display units, which could project one of 12 two-dimensional stimuli on its 2 in $\times 1 \frac{1}{2}$ in display screen. Below each of the outside display units was a lever. Reinforcement consisted of a peanut-flavored pellet. The animal was seated in a chair. Water was available at all times.

The Primate Automatic Testing Apparatus-Key (PATA-K) was designed by the Holloman AFB Comparative Psychology Laboratory. It used an identical display as the PATA-L, but instead of responding to a lever, $S$ pushed the display panel. The panel was a side of the animal's cage.

\section{Problems}

A two-position oddity problem was used. The center position was never odd. The WGTA stimuli were tridimensional wood objects, made as similar as possible to the in-line displays.

Two types of problems were used: 6-trial and 1-trial oddity. In the 6-trial problem animals received the same oddity problem for six consecutive trials, position of the odd object varying randomly. In the l-trial problem, a given oddity problem never appeared consecutively.

The testing session consisted of 48 trials and was generated in the following manner for all apparatus. Nine of the 12 stimuli were used to generate the oddity problems. This allowed for 72 oddity problems. Six series for both the 6-trial and l-trial group were randomly determined. Position of the odd object was determined randomly with right-left choices being evened out for each series.

A test series was constructed by pairing the three remaining stimuli with stimuli already used and with each other. This test series was used after the l-trial problem had been solved to criterion.

\section{Procedure}

6-Trial Oddity: Animals were run either once or twice a dayk with a minimum inter-session time of $4 \mathrm{hr}$. A non-correction procedure was used. Six-trial animals were run until they attained a criterion of $90 \%$ correct for three series. Time to complete each series was recorded. Each animal was then shifted to the 1-trial oddity problem. Upon attaining criterion, he was given the test series.

1-Trial Oddity: With the exception of the number of trials per problem, this group was treated exactly as the 6-trial group.

Transfer. After attaining criterion on 1-trial oddity and receiving the test series, certain animals were transferred to a new apparatus. All pre-training procedures used for each apparatus were employed, and when completed, an animal began work on the appropriate oddity problem.

\section{Results}

Comparison of Apparatus. The three types of apparatus were compared on three measures: session to criterion, mean time per session, and mean errors per session. The data were subjected to three separate analyses of variance. There were no differences between the 1-trial and 6-trial problems on all measures. The only significant difference between apparatus was on the time measure.

A $t$ test suggested that the PATA-K may be superior to the WGTA and PATA-L combined $(t=2.39)$ and approached the .05 level of significance when compared to the WGTA $(t=2.02)$. Nevertheless, it must be remembered that the apparatus effect per se did not yield a significant $F$ ratio.

Transfer. Table I shows the amount of transfer when animals were switched from 6-trial problems to 1-trial problems and from one apparatus to another. Animals showed significant 6-T, 1-T transfer, although it was far from perfect, but showed, on the average, no indication of apparatus transfer. This conclusion is strengthened by an insignificant negative rank order correlation (Rho=-.14) between the trials to criterion

Table 1. Mean Sessions to Criterion as a Function of Transfer

$\begin{array}{lrllccc}\begin{array}{l}\text { Transfer } \\ \text { Problem }\end{array} & & \text { N } & \begin{array}{c}\text { Original } \\ \text { Learning }\end{array} & \begin{array}{c}\text { Transfer } \\ \text { Learning }\end{array} & \begin{array}{c}\text { Mean Sav- } \\ \text { ings Score }\end{array} \\ \text { 6T-IT } & \overline{\mathrm{X}} & 7 & 68.7 & 36.7 & +47 \% \\ & \text { SD } & & 50.6 & 26.4 & \\ \text { Apparatus } & \overline{\mathrm{X}} & 12 & & 73.2 & 74.7 & -2 \% \\ & \text { SD } & & 41.3 & 64.7 & \end{array}$




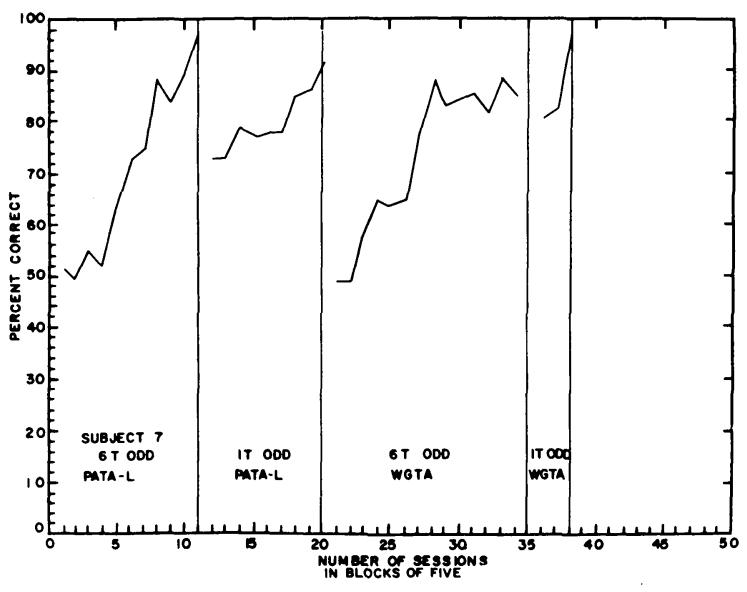

Fig. 1. Typical transfer curve for a 6-trial animal.

on the original problem and the transfer problem. Apparatus transfer was tested in all possible directions except for transfer from WGTA or PATA-L to the PATA-K.

Figure 1 shows a typical learning curve for a 6-trial animal to a 1-trial problem and his transfer to another apparatus.

\section{Diseussion}

The study most similar to this study was done by Levine \& Harlow (1959) investigating 1-trial and 12-trial, 2-position oddity. In 3024 trials, the Levine and Harlow 1-trial animals attained at $70 \%$ level of responding. In the present study, the average number of trials for the WGTA group was 3952 to a $90 \%$ level. This suggests that the present WGTA results are typical.

The two automatic modes of presentation (PATA-L and PATA-K) are no more difficult for Rhesus monkeys than is the WGTA mode. This is somewhat surprising since studies have indicated that stereometric object discriminations are easier for monkeys than planometric object discrimination. (Harlow, 1945).

In considering the trials per problem dimension, Levine used a 12-trial problem consisting of 12 consecutive presentations of objects $A$ and $B$, with either $A$ or $B$ odd. In the present study, during a 6-trial problem, the same object was always odd. Thus, animals could solve the problem on an object quality discrimination basis or oddity. Apparently the procedure of the present study ruled out the type of systematic error which Levine and Harlow found to depress the 12-trial group performance. The transfer data from 6-trial to 1-trial problems suggests that the animals were using both oddity and object quality discrimination hypothesis and that additional trials were needed before the oddity principle was firmly grasped.

The results of the apparatus transfer were unexpected and surprising. It was felt that the animal's responding to the test series at a criterion level was a good indication that the oddity principle was firmly grasped. However, the failure to show transfer indicates that monkeys do not have an oddity concept independent of the stimulus-response configuration associated with each apparatus and test situation. These results, and follow-up studies with chimpanzees and humans now in progress, lead us to believe that there are two types of transfer. One involves learning task $\mathrm{A}$ in situation $\mathrm{A}$ and then applying exactly the same principle to situation B. The other is learning task $A$ in situation A, and then learning task $B$ in situation $A$ in which task $B$ has certain elements common to task A. We now have data that indicate monkeys do show the second kind of transfer while children show both types.

\section{References}

Rohles, R. H., Belleville, R. E., \& Grunzke, M. E. Measurement of the higher intellectual functioning in the chimpanzee. Aerosp. Med., 1961, 32, 121-125.

Harlow, H. F. The formation of learning sets. Psychol. Rev., $1949,56,51-65$.

Levine, M., \& Harlow, H. F. Learning sets with one- and twelvetrial oddity problems. Amer. J. Psychol., 1959, 72, 253-257.

Harlow, H. F. Studies in discrimination learning by monkeys: V. Initial performance by experimentally naive monkeys on stimulus object and pattern discrimination. J. gen. Psychol., 1945, 33, 3-10.

\section{Notes}

1. This work was supported under Air Force Contract No. 29(600)2418. Grateful acknowledgment is made to the Comparative Psychology Laboratory personnel at Holloman AFB for their cooperation and encouragement.

2. A more complete copy of this report is available from the author upon request. 